\title{
Problems and Challenges for the First Generation Learners
}

\author{
Shakibur Rahman Khan* \\ Prince Sattam Bin Abdulaziz University, Kingdom of Saudi Arabia
}

Corresponding Author: Shakibur Rahman Khan, E-mail: shakibkhan.khan5@gmail.com

\begin{abstract}
ARTICLE INFO
Article history

Received: June 10, 2017

Accepted: August 21, 2017

Published: December 01, 2017

Volume: 6 Issue: 7

Special Issue on Language \& Literature

ABSTRACT

The article Problems and challenges for the First Generation Learners seeks to explore the important issues and problems encountered by first generation English learners and teachers in the context of learning and teaching in Saudi Arabia. It is assumed that the quality of teaching, teachers, and teaching materials provided to the first generation learners as in the context of my experience of teaching, Wadi Ad-Dawasir, Sattam Bin Abdul-Aziz University, has to have certain standards and measuring rods to assess the impact of teaching.
\end{abstract}

Advance access: September 2017

Key words: Learners, Environment, Fear Factor, Acclimatization

Conflicts of interest: None

Funding: None

\section{PROBLEMS AND CHALLENGES}

Teaching English to first generation learners need a more fundamental understanding of the exact nature of the problems and its complexity of dimensions like course designers, teachers, learners and concerned educational institution. This is because of a variety of reasons. Thus, second, or foreign language learners, teachers and syllabus designers' responsibilities are complex in nature. A learner, teachers and syllabus designers start from scratch. A learner needs a good model for listening, speaking, reading and writing.

"A first generation student is one whose parents or guardians have not attended a post-secondary institution. If your sibling has attended a College or University, but your parent(s) or guardian(s) have not, you are still considered a first generation student".

Usually in the first generation learning and teaching, the majority if not all the teachers are not capable or qualified to teach English. They are very weak at all levels. They are not good models for learners in linguistic aspects or pedagogical. In addition to their weakness in English language, they are not given adequate training in different approaches, methods and techniques of teaching the two languages especially in the textbooks that are taught for the first generation learners.

The syllabus designer should introduce the passage with the grammatical structure of the sentence before embarking on the reading of the passage. The grammatical structure should also be simplified as follows: the sentence is a complex one. It involves two clauses. One is the main clause and the other one is the subordinate clause. The subordinate clause functions as an element of the main clause i.e. as an adverbial of the main clause expresses the time.
"Ahmed (Subject of the main clause) writes (the verb of the main clause) a story (object of the main clause) whenever he visits his grandfather(subordinate clause, adverbial of time, it can be replaced by "this time") in the village (adverbial of place, it can be replaced by here)".The sentence can be rephrased as:

S1.Ahmed writes a story now and here.

So, it is obvious that "whenever he visits his grandfather" is a replacement of "now" and "in the village" is a replacement of "here".

The subordinate clause is also consists of Subject/pronoun $(\mathrm{He})$ a verb (visit $+\mathrm{s}$ (third person singular S.) object (his grandfather) and adverb of time/whenever $=$ at the time transformed to the initial position to join the main clause to the subordinate clause (subordinate conjunction.

Some specialists would say that the learner would be burdened by these terminologies and the explanation of the structures. This will make it more difficult for the leaners. I would say that the first generation learners in our situation learn the language and the structure of language consciously. A learner produces a sentence consciously on the analogy of the structure of another sentence. For him there is no choice but to learn the structure of a sentence and construct analogues ones consciously because unconscious internalizing and production of a structure of a sentence does not happen with the first generation learner and at least not in our context where the exposure to language is very limited. However, the teacher need not burden the learners with the terminology. He can summaries the two structures comparatively by juxtaposing the two structures side by side:

Ahmed writes a story/whenever he visits his grandfather/ in the village/. 
Ahmed writes a story/now/and/here/.

Then, he should ask the learner to produce analogous sentences by providing $\mathrm{S} 1$ and ask the learner to make S2.and the vice versa is also true.

The phonetic symbols of the pronunciation of words are to be furnished with for the teacher who has no time to refer back to a pronunciation dictionary. It will also reduce the effort exerted by the leaner that has to look up the pronunciation of words in a dictionary such as happy/'hæpi/.This process of repetitive search for the pronunciation and meaning of words would waste huge amount of time and affect the interest of a learner. Ideally for the first generation leaners a text book has to be accompanied with a CD and a cassette so that the teachers of the first generation learners themselves have to have opportunity to listen to before teaching and also the first generation learners should have the opportunity of listening to the words articulated separately and the text read repetitively to compensate for a family members. The reading passage is to be followed by exercises on the grammatical structures(different structures have to be presented to express the same point graded from simple to complex structures), meaning, prepositions, and usage have to be covered in the exercises. Class is divided into groups to give opportunity to everyone to read loudly in each group and the teacher room around the groups to verify the performance of the individual. These are followed by comprehension questions to verify and guaranty that the learners understood thoroughly the content of the paragraph. In each reading paragraph syllabus designers should pin point the cultural issues presented in a text to raise the awareness of the teachers and the learners towards the cultural issues in the text. The learners should be asked to frame sentences analogous to the patterns and structures that are previously stated on their own. This step will be introduction to teaching writing a same paragraph on their own. This followed by free writing on similar topic. All these steps are presented in a unit of teaching.

So, it is not a second language. English should be taught as a foreign language. Still the specification of the aim should be clearly stated. In addition to the status of English as a compulsory subject, it is the language of instruction in some disciplinary fields, such as Engineering, medicine, computer, nursing, pharmacology, and many other disciplines. English is important for contacting the wider world. It is a library and airlines language. English is the first language for many dominating counties such as USA,UK, Australia, New Zealand, Canada, and South Africa. It is also the second language of many countries and a foreign language for the rest of the world. People from Arab countries can access their thoughts, beliefs, ideas, culture, literature, opinions, and attitudes in English with the people of rest of the world. It helps to avoid cultural clashes and misunderstanding between nations.

English is a library language. It helps to acquire a thorough knowledge from all branches of study. The majority of world scientific journal, newspapers, periodicals, results of research centers etc. is brought out to the world in English. Consequently, to keep oneself abreast with the new scientific theories, technological development, and pursue higher education, English is necessary.
Hence, it is obvious that we do not need thorough knowledge of English in all walks of life. We need thorough Knowledge of English in certain domains. However, in these certain domains we need a very highly qualified and competent English users who know accurately, and proficiently the fourfold English language skills to be able to carry out a very effective discussion, translation of different texts and contexts, and a reciprocal exchanging of a very complex technical and discourses. This necessitates learners of a very good characteristic, a very good qualified teacher, an excellent teaching materials and well defined aim, and enlightened administrative staff. It is crystal clear that only some of the current learners enjoy the necessary characteristics of good learners, only very few qualified teachers, and rarely to find excellent teaching materials, with clearly specified aim, and enlightened administrative staff. The work is a complementary.

Brookfield, (1993)suggests the following strategies to achieve that:

"Value and respect participants through word and action. Listen attentively and provide support for efforts. Identify and challenge assumptions. Reflect back attitudes, rationalizations, and habitual behaviors. Imagine and explore alternatives. Practice reflective skepticism. Model critical thinking through clarity, consistency, openness, and accessibility. Teach theory by detailing phenomena and practical problems related to the theory. Balance conceptual with concrete information. Use a variety of sketches, plots, schematics, computer graphics, and physical demonstrations in addition to oral and written explanations in lectures and handouts. Provide time for participants to reflect upon the material being presented. Encourage active participation."

He should vary his presentation methods approaches techniques and individualize his strategies. He has to address skills, for example, by role-play lectures using activities of translation and interpretation, writing a paragraph, discussion etc. by the learners.

He should use different channels to impart knowledge by multiple means including lectures, handouts, videos, analyzing case studies, sharing of personal experiences, and discussion. He should use different intelligences to reach his objectives, forexample, Logical and linguistic intelligences are used in traditional academic settings considerably.This was responsible for high scores in academic achievement tests.

The most important characteristics of a learner that can play an important role in the learning and teaching speed process can be enumerated as follows: Intelligence, personality motivation of different types attitude, aptitude, style of learning, age, intuition of different aspects, identity and group affiliation, learner religion and beliefs, rate of learning, learning skills, confidence, extroversion, introversion, social setting, educational ambiance etc. Again under each of these categorical labels other sub classifications can be further detailed. It is assumed that the success in language learning is correlated with degree or intensity of the categories previously stated. 


\section{The Third Factor}

It a common knowledge that teaching and learning is a construction based on a foundation of contributing components: learners, teaching materials, teachers, clear specific aims and objectives, and enlightened administration. Specific aims is not less important that any of the rest of the pillars because the process of teaching is directed to a certain orientation. Like a plane has to embark from certain location and directed straight forward to a specific destination/aim without any deviation from the path. Otherwise the journey will end with catastrophe. Similarly, teaching process depends on to what extent the aim and objectives were clear and straight forward. Hence, the aims and objectives on which basis the characteristics of the teachers, learners, teaching materials, administration should be selected must be very clear and straight forward. Exactly the design makers state clearly the quality of the product that they want to the administration and the administration in its turn provide the raw materials/ learners and request the experts/teachers to produce a product of certain quality/scholars. The quality of the targeted product should be very clearly described and prescribe in order to juxtapose it in comparison with the quality of the produced product/scholar to verify how far the quality of the produced product correlate with the targeted one to measure the success or failure of the producer/teacher and consequently evaluate the appropriateness of the interfering factors interfering factors (teaching materials, the role of the administration and the ambiance).Now, if the product of the schools and universities graduates evaluate the gap between the expectations and the quality of the real productions will be very deep.

Psychologist Benjamin Bloom developed a classification scheme for the types of learning which includes three overlapping domains: cognitive, psychomotor, and affective. The important one for us as a language pedagogics and teachers is the cognitive learning/knowledge which is the formal term used for mental skills such as recall of information. Carole Lee (1999) presents five important points in the skills in the cognitive domain. These are the following:

- "Knowledge(remembering information);

- Comprehension (explaining the meaning of information);

- Application (using abstraction in concrete situation;

- Analysis (breaking down into component parts);

- Synthesis (putting parts together to form a new and integrated whole)."

To apply these to language learning one can say that knowing the rules of constructing simple English sentences: Subject + verb compliment is knowledge. Explaining the meaning and implications of this rule is comprehension. Application is illustrated and achieved by constructing and deriving the correct English sentences on the basis of the stated rule.

All the three very wise Saudi students have graduated lately.

Analysis is necessary for analyzing the component parts into immediate constituents; namely, Subject/NP(all the three wise students) + Verb phrase(have graduated) + adverb (lately). The subject/NP can be analyzed further into the following:

Predeterminer (the) + central determiner (the) + post-determiner (three) + adjectival phrase premodifier (very wise) + adjective premodifier (Saudi) +the head of the NP (student)+ Plural ... This finally followed by synthesis which is the amalgamation of the components into a whole pattern unit.

These are very crucial ability for language learners

1. "Logical-mathematical intelligence: the ability to detect patterns, think logically, reason and analyze, and compute mathematical equations (e.g., chemists, economists, engineers).

2. Linguistic intelligence: the mastery of oral and written language in self-expression and memory (e.g., journalists, lawyers, politicians).

3. Spatial intelligence: the ability to recognize and manipulate patterns (large or small) in spatial relationships (e.g., architects, pilots, sculptors)

4. Musical intelligence: the ability to recognize and compose musical quality (pitches, tones), and content (rhythms, patterns) for production and performance (e.g., composers, conductors, musicians).

5. Kinesthetic intelligence: the ability to use the body, or parts of the body to create products or solve problems (e.g., athletes, dancers, surgeons).

6. Interpersonal intelligence: the ability to recognize another's intentions, and feelings (e.g., managers, sales people, social workers).

7. Intrapersonal intelligence: the ability to understand oneself and use the information to self-manage (e.g., entrepreneurs, psychologists)."

The tenor of Gardner's theory is that people use the above seven types of intelligence according to the necessary type of learning, individual strengths and capability, and the focus of the interaction.

\section{Auditory Learners}

There are three main types of learning styles: auditory, visual, and kinesthetic. Most people learn best through a combination of the three types of learning styles, but everybody is different. Auditory Learners rely non hearing. He would prefer listening to an explanation rather to reading. Verbal language is the prime form for exchanging information. This type of learner is very good in learning oral language. Generally they read slowly and have trouble writing, struggle to follow written directions. They learn best through the following techniques:

1. Play word games and use rhymes to practice language.

2. Have the child read aloud, even when alone, and follows the text with his finger.

3. Allow the child to explain concepts verbally and give oral reports.

4. Have the child memorizes information by repeating it aloud.

5. Assign projects and study times to be done in small and large groups.

6. Read aloud often to young children. 
7. Provide a personal voice recorder the child can use to record notes or questions.

8. Use beats, rhythms, and songs to reinforce educational information

\section{Visual Learners}

The best way to teach visual learners is by demonstrating things to learners and reading. They absorb information by seeing it in front of them and storing the images in their brains, often enjoy reading, have good handwriting, are very detail-oriented, are organized but they may have difficulty focusing while listening to an explanation and tend to struggle with verbal directions and are easily distracted by noise. They often need to maintain eye contact with a person to concentrate on a conversation. The following are suitable tips to help these types of learners.

- Write out directions.

- Use visuals when teaching lessons, such as pictures, charts, diagrams, maps, and outlines.

- Physically demonstrate tasks.

- Use visual aids such as flashcards and blocks.

- Show the visual patterns in language to teach spelling, vocabulary, grammar, andpunctuation.

- Organize information using color codes.

- Talk with the child face-to-face and make eye contact whenever possible.

- When directions are given verbally, encourage the child to ask for clarification when she/he doesn't understand fully.

- Encourage the child to write plenty of notes and organize information on paper and with objects.

- Provide a quiet, neat place to study, and minimize distractions as much as possible.

Although most people use a combination of the three learning styles, they usually have a clear preference for one. Knowing and understanding the types of learning styles is important for students of any age. It is advantageous for students to understand their types of learning style early on so that homework and learning may become easier and less stressful in the future. Although it may be tempting to stick with what works, it's important to practice and train the other types of learning styles early on so that, as he grows, the child can utilize the other types just as effectively.

What is so called "A Renaissance Child" A well-balanced, intelligent child is able to develop a combination of all three types of learning styles in spite of the fact that he has preference for one. A child has a dominant learning style and can improve other types. Relying on one dominant style only can debilitate the learners 'potential. The most important learning styles that need to be trained are the cognitive skills. Cognitive skills are the foundational building blocks of each learning style. Without properly trained cognitive skills, a child isn't able to use or take advantage of the other learning styles effective.

How is learning processed?

According to Martin Rayala (1999) Learning occurs in four means; namely, transmission, acquisition, accretion and emergence.
"Transmission is the process by which information; knowledge, ideas and skills are taught to others through purposeful, conscious telling, demonstration, and guidance. Over the course of a lifetime, this method accounts for only about $10 \%$ of learning. Unfortunately, this is the most traditional and, currently, the most predominate method of instruction. However, we are finding out it is not very effective and moving toward acquisition and emergence, and examining the lessons of accretion.

- Acquisition is the conscious choice to learn. Material in this category is relevant to the learner. This method includes exploring, experimenting, self-instruction, inquiry, and general curiosity. Currently, acquisition accounts for about $20 \%$ of what we learn.

- Accretion is the gradual, often subconscious or subliminal, process by which we learn things like language, culture, habits, prejudices, and social rules and behaviors. We are usually unaware that the processes involved in accretion are taking place, but this method accounts for about $70 \%$ of what we know. Social learning certainly plays into this type of learning, as does the hidden or covert curriculum.

- Emergence is the result of patterning, structuring and the construction of new ideas and meanings that did not exist before, but which emerges from the brain through thoughtful reflection, insight and creative expression or group interactions. This form of learning accounts for the internal capacities of synthesis, creativity, intuition, wisdom, and problem-solving. This method is greatly dependent on the allocation of time, and opportunities to reflect and construct new knowledge. This method plays an important role in inspiration and originality. In the context of current educational practices, we learn only $1-2 \%$ by this method.

- In the context of these definitions, examine your own learning experiences and teaching styles.

\section{CONCLUSION}

Wadiaddawser is a countryside serving as a conduit between Riyadh and Abha has the first generation learners tossed and frightened to be exposed to a language that has utility for them in terms of an exposure that is too abrupt.Understanding the relationship between individual differences of the teachers and learners, variables in teaching materials, differences in social situations, enlightened flexible administration and success in language learning is very important issue in language teaching. All these factors have to be activated and interacted in a complete harmony because they all interplay in a variable percentage in the achievement of an excellent or bad product. Many other educationists may not agree with this opinion; however, the experience of the researcher concludes that there are a strong association between the aforementioned factors and a qualitative educational result. It is agreed that there are no clear cut yardstick of measuring the role of each one of these factors but any experienced teacher and a successful learner must give the priority of importance to the learner's characteristics in language learning and teaching process. It is agreed that a sensitive teacher can 
change the equation and percentage of correlation between the contribution of other factors, but also here it is indirectly implied that there is enlightened administration which gives incentives to the teacher to exert extra efforts and do not interfere in the experts work. An example may clarify the issue. A good qualified competent sincere teacher would not be able to change the situation if, for example, the learners aim only at getting a certificate by hook or by crook without exerting due efforts that is done by a counterpart at the national or international standard. The immature learners may create problems for this type of a teacher to avoid the needed hard working. Language learning is ideally achieved by a lot of efforts on the part of the learners. They should practice and practice as often as possible. We should not forget a very important issue in language teaching and learning which the difficulty is emerged from the culture and nature of the mother tongue. Here, the role of a syllabus designers and teachers is very important to facilitate the learning and teaching process. They have to maximize the efforts and the guessing of the learners by tackling the issue of language differences and the common errors that encountered the learners. The course designing must be done according to the most renowned theories approaches, methods and classroom teaching experience and researches. Taking into consideration all these factors and opinions will undoubtedly yield a fruitful success and improve the language learning and teaching. A qualified teacher should be acquainted with different types of learners, different styles of learning, different types of knowledge, types of learning and the method, approaches and techniques for teaching every type. He has to be able to identify each type of learners in order to appropriately deal with.

\section{REFERENCES}

Lightbown, P. M., and Nina, S. (2006). How Languages are Learned,Oxford Handbook for language Teaching Oxford University Press.

Brookfield, S. (1983).Adult Learners, Adult Education and the Community.Open University Press.Milton-Keynes; UK.

Swanson, H. L., Maureen, H., and Carole, L. (1999). Interventions for Students with Learning Disability, A meta-Analysis of Treatment Outcomes, The gullford Press.

Tennant, M. (1995), Types of Learning-University of Washington, (htt://www.Washington.edu/doit/TeamN/ types.htm/.

Fry, H., Ketteridgesteve, C., and Stephanie, M. (2009),A Handbook for Teaching and Learning in High Education: Enhancing Academic Practice,Routledge, New York and London.

Greenbaum, S. (1996). The Oxford English Grammar. Oxford University Press Inc. New York. 